\title{
Design and manufacture of artificial organs made of polymers
}

\author{
Marek Macko" ${ }^{1}$, Zbigniew Szczepański ${ }^{1}$, Dariusz Mikołajewski ${ }^{1}$, Joanna \\ Nowak $^{1}$, Emilia Mikołajewska ${ }^{2}$, Jacek Furtak ${ }^{3}$, and Stawomir Listopadzki ${ }^{4}$ \\ ${ }^{1}$ Faculty of Mathematics, Physics \& Technical Science, Kazimierz Wielki University in Bydgoszcz, Poland \\ ${ }^{2}$ Department of Physiotherapy, Ludwik Rydygier Collegium Medicum in Bydgoszcz, \\ Nicolaus Copernicus University in Toruń, Poland \\ ${ }^{3}$ Department of Neurosurgery, The 10-th Military Research Hospital, Bydgoszcz, Poland \\ ${ }^{4}$ University Hospital no. 2 in Bydgoszcz, Department of Laparoscopic Urology, Poland
}

\begin{abstract}
New technologies such as 3D printing and reverse engineering are becoming increasingly popular, including in medical applications. The paper presents possible ways to use 3D printing in the area of increasing the level of medical training specialists in their daily clinical practice and in the selection of the appropriate scenario before a real surgical operation. Descriptions of the projects carried out with the surgeons aimed at achieving the aforementioned goals were presented. The presented concepts are relatively new solutions, but their further development may lead to the extension of the field of application of these techniques in medicine, among others in relation to other specialties.
\end{abstract}

Keywords: reverse engineering, artificial 3D organs, personalized medicine

\section{Introduction}

The applications of traditional design and manufacturing for the purposes of daily clinical practice have many limitations, eg slow preparation and personalization of products. Traditional education based on the anatomy of human corpses depicting the natural variation of anatomy has limitations due to the ubiquitous education of medical specialists. New technologies such as three-dimensional (3D) printing and reverse engineering [1-6] can be used to solve the above problems. New technologies can increase the level of traditional teaching due to lower costs, scalability, diagnostics and object learning, covering the entire age range, gender and can be used all over the world [7-11]. In addition to their educational character, they are of great importance at the stage of analyzing the geometric features of a particular patient and using this data to create an artificial organ model in 3D printing technology. Artificial organs prepared in this way are used during the preparation of the proper surgery scenario.These technologies can be considered new and

\footnotetext{
* Corresponding author: mackomar@ukw.edu.pl

Reviewers: Piotr Krawiec, Kateryna Kravchenko
} 
complementary, but they can also support current applications of medicine in everyday clinical practice. They can also give rise to a new family of commercial techniques that optimize 3D-based technologies for 3D printing in a fully functional clinical solution. It should also be mentioned that computer aided methods are used in various fields of medicine, such as in cardiology, dentistry, but also in the field of telemedicine applications [12-14].

\section{Methodology}

Data for work (MRI and CT) have been transferred as files in the Dicom standard. Magnetic resonance imaging (MRI) uses electromagnetic radiation produced by radio waves harmless to the body, while in computed tomography (CT), the energy carrier is $\mathrm{X}$ waves or X-rays, which too often or too high doses can lead to DNA damage. For the processing of data (raster graphic images), and then creating 3D objects and their editing, several applications were used and proprietary sub-applications were implemented. One of the programs used during the project implementation was the Simpleware ScanIP application. It is a software for visualization and 3D image processing. ScanIP provides a programming environment for comprehensive 3D image data processing (MRI, CT, micro-CT, FIB-SEM, etc...). The software offers powerful tools for visualization, analysis, segmentation and image quantification. The ScanIP application includes a number of video recording functions and options for exporting surface / mesh models from data segments for $\mathrm{CAD}$ and $3 \mathrm{D}$ printing. Additional modules were used to export CAE mesh, integrating image and CAD data, exporting NURBS and calculating effective material properties during scanning. A number of works have been carried out to improve the manual segmentation of low-contrast medical data using a multi-layered surface grid and then to generate STL files.

In order to assess the suitability of available 3D applications for the needs of the project, *.stl models were imported and edited in following applications: InVesalius 3.1, ScanIP, MeshLab, Netfabb Basic, Blender and SolidWorksFast, layered melting and solidification of the material during the process, does not lead to rhenium gravitational sedimentation. Therefore, Inconel 718-Rhenium alloy can be successfully process with the use of SLM technology, and it should perform better in elevated temperatures, in comparison with standard Inconel 718.

For the needs of the work, patient's data were obtained according to two methods: Computed Tomography (CT) and Magnetic Resonance Imaging (MRI). After analyzing the data obtained from scans, the (MRI) method proved to be more useful. The method (CT) is focused on precise tissue analysis with regard to higher density than bone tissue. This is due to the use of X-rays. X-rays represent a larger spectrum of densities, although smaller densities are more difficult to reproduce and are much closer to each other in e.g. the 8-bit data structure obtained from the scan. The method (MRI), because of magnetic resonance imaging, better illustrates soft tissues containing more atoms such as iron, etc., whose aggregations (agglomerations) are very well recognized in this method. The method (MRI) thanks to this, has a much larger spectrum of selection of the density threshold during soft tissue scan, in this case the threshold is much easier. The data obtained in this way was processed in the InVesalius 3.1 application to form solid objects, and then edited (Fig. 1). 


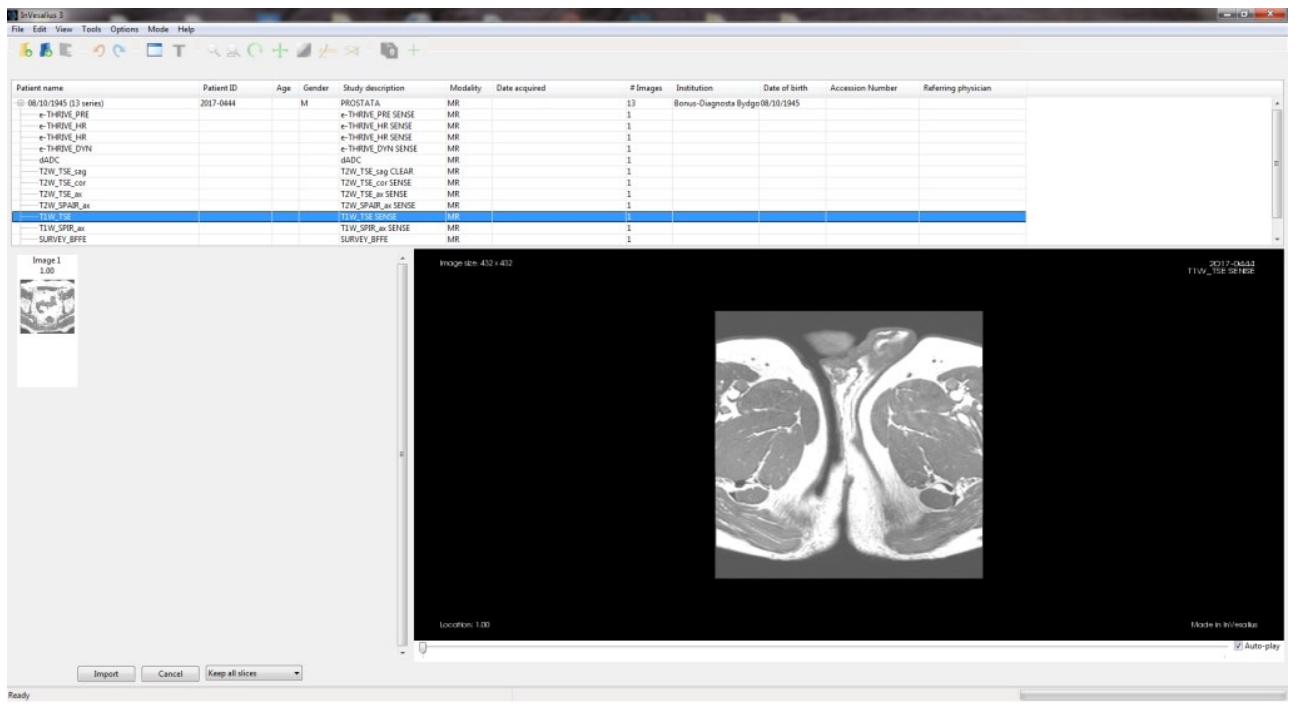

Fig. 1. InVesalius screen view with an enlarged patient prostate gland

During the tests, data obtained from both patients with contrast and without contrast were used. The contrast proved to be very useful from the point of view of $3 \mathrm{D}$ analysis provided it was applied directly to the organ to be analyzed in the case of 3D scanning. The process of 3D scanning is not understood in this case, as a direct recognition of the structure, and is a series of treatments that allow obtaining data analogous to the $3 \mathrm{D}$ scan, which are the basis for creating a stereophotographic structure, which is the basic standard of $3 \mathrm{D}$ geometry in further procedures allowing the creation of an organ replica. To obtain and correctly interpret data, consultations with CT and MRI scanners were necessary on average once every two weeks.

The main standard used during work with medical installations was the DICOM standard. It is treated as a medical standard and contains a number of additional information about the patient's condition, however, for the purposes of the project, the focus was only on geometric data obtained in the form of cross-sections, which were later changed into three-dimensional matrices. Data obtained in other standards, including JPG, BMP and STL directly from the scanning machine software, or indirectly, can also be used in the implementation of the project. Data from the above-mentioned standards must be interpreted as 3D matrix data. The effect of these works are spatial objects presented in various applications.

The Mimics application was also used, which enabled importing from DICOM and other files (JPEG, TIFF, BMP, x-ray) graphics necessary for creating and editing files in the 3D standard and for creating necessary FEM simulations and preparing for printing in 3D printing standard.

In the environment of 3D application users, the Blender application is very often used. There are free and open software for modeling and rendering images and three-dimensional animations with an unconventional user interface. In own work, an application was used to create and later edit various 3D graphic standards, such as: meshes, NURBS surfaces, Bézier curves, meta objects, vector fonts (TrueType, Post Script, OpenType). The application was also used for "net sculpting", catmull-clark division of grids, Boolean functions for grids, mesh editing based on vertices, edges and polygons (polygons), Python scripts support, with which you can add new editing tools, BMesh - a system that allows you to 
create and edit walls with a very complicated structure - for example, about a dozen or so edges. Implementation of the mentioned applications and own modifications led to the creation of files that are directly sent to the 3D printer. Prints were made, successfully completed, selected organ geometries that will be used to make the mold for a model made of material resembling the structure and properties of the source organs.

Taking into account the conclusions of the work carried out and the overall assessment, it can be concluded that for an efficient process of editing and preparing the object for 3D printing, it is necessary to use several applications, but the application of InVesalius 3.1 is the most important. The next step was to adapt the 3D printing technology to ensure the expected geometric accuracy. For economic reasons, the use of FDM, SLA and/or PolyJet printing technologies has been proposed. The FDM technology uses thermoplastic material, which is brought to the plastic state is applied with a thin stream on subsequent layers of the object. The project uses a Gaja 3D Multitool printer. The SLA technology uses acrylic resin, point hardening laser beam. The project uses a Nobel 1.0 printer. PolyJet 3D printing technology (OBJET 30 pro printer) was not used due to the high cost of the material (acrylic resin). All mentioned 3D printing technologies (FDM, SLA, PolyJet) are in possession of the Laboratory of Technical and Medical Prototyping located at the Institute of Applied Mechanics and Computer Science of the Casimir the Great University in Bydgoszcz.

The next stage was the preparation of specific models for printing, which required generation of the print head / path in both FDM and SLA technology. The user interface of an application that supports FDM - Kisslicer printing is presented below.

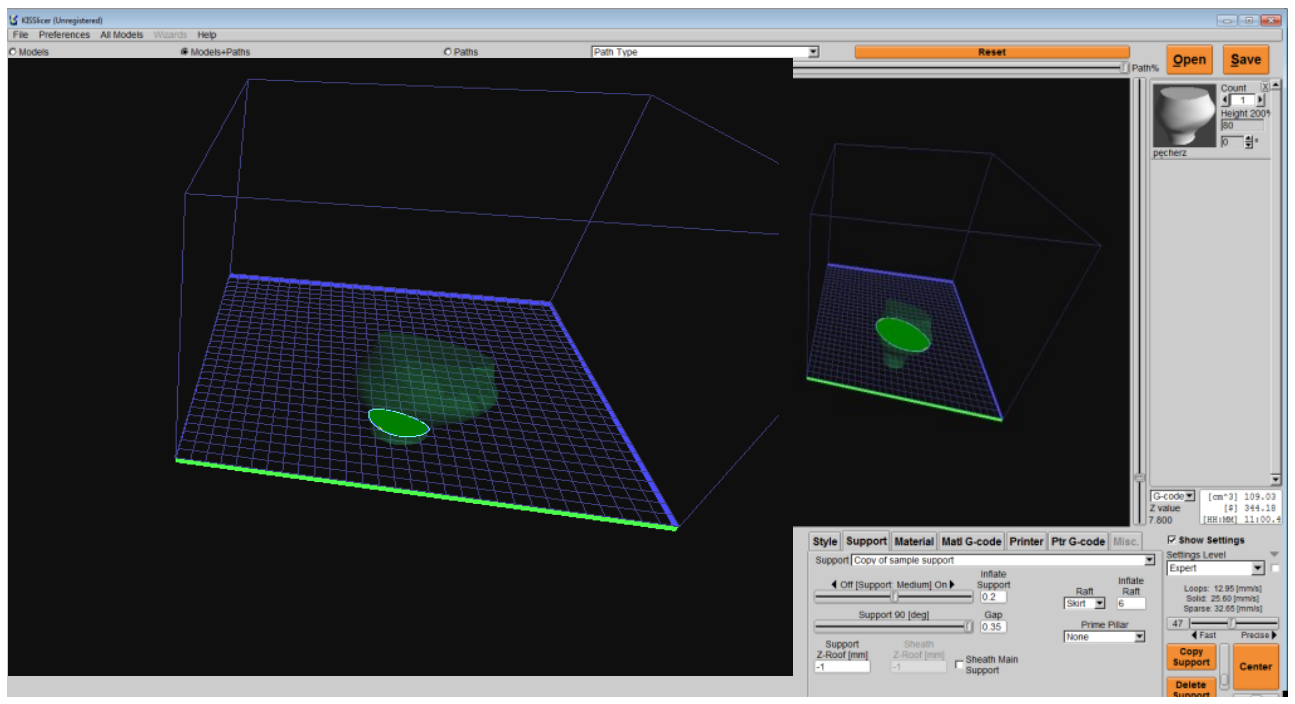

Fig. 2. Kisslicer application interface for setting a 3D printer working in FDM technology for printing (solid model inserted in the virtual printer chamber)

The Fig. 3 shows the successive stages of creating an artificial organ by the following stages: obtaining an image from a patient's specific organ tomography, processing and editing a 3D model, visualization of an organ in 3D technology, printing on a $3 \mathrm{D}$ printer. 


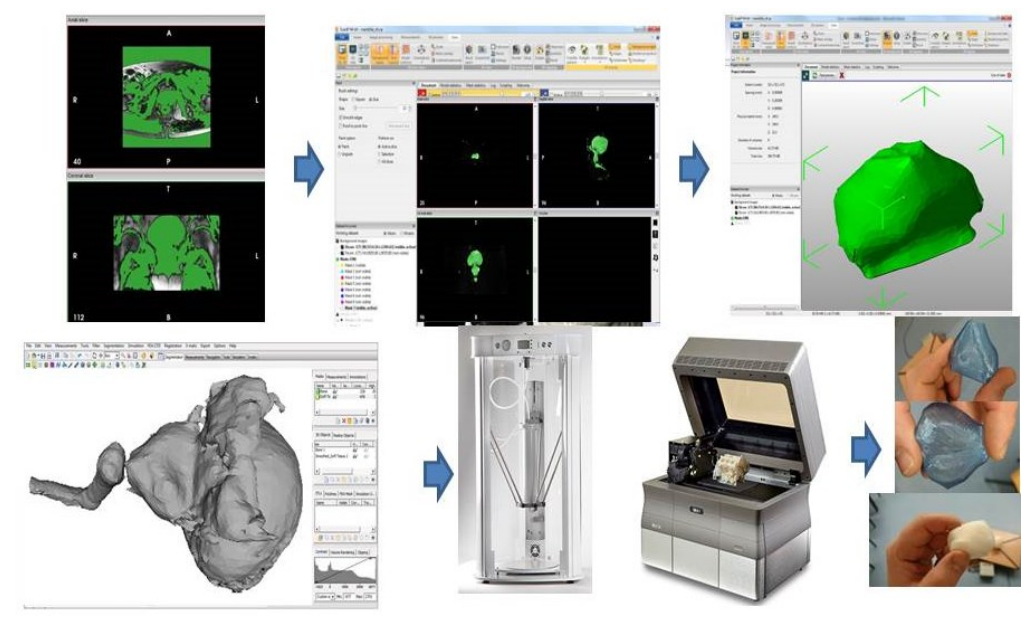

Fig. 3. Following stages of creating an artificial organ: obtaining an image from a patient's specific organ tomography, processing and editing a 3D model, visualization of an organ in $3 \mathrm{D}$ technology, printing on a $3 \mathrm{D}$ printer

\section{Experimental results and discussion}

The process of preparing this type of medical organs is complicated and it is carried out in several steps, and the final result depends on the software used and the user's skills. The MeshLab and GOM Inspect application analyzed the devia-tions of the STL file for 3D printing from the mesh model with DICOM. Flat masks have been identified that allow the separation of zones that mark bones from soft tissues. This was accomplished by designating a range of gray threshold values. After applying the masks and extracting specific areas and creating a three-dimensional surface model, it was exported to the STL file. At this stage, it was possible to further process the obtained net form, which still contained many errors and could not be the basis for the 3D printing. In order to clean and repair the mesh model, applications were used: MeshLab and GOM Inspect. Based on them, errors in the structure of the mesh geometry were removed: artefacts and errors created during the conversion of a series of DICOM images into a spatial mesh model. Fragments of the grid lost at the stage of converting DICOM images into a mesh model and during its correction were supplemented with the use of the free version of the GOM Inspect program. One of the first successful attempts to cooperate with surgeons was the task of obtaining the object, editing and printing the patient's skull, with particular attention to the nasal septum for illustrative purposes, before performing the nose correction surgeons otolaryngologists. The printout of the nasal chamber made of polymer materials was used for a detailed analysis of the operation of the nose, taking into account the specific structure of the nasal passages (fig. 4). 

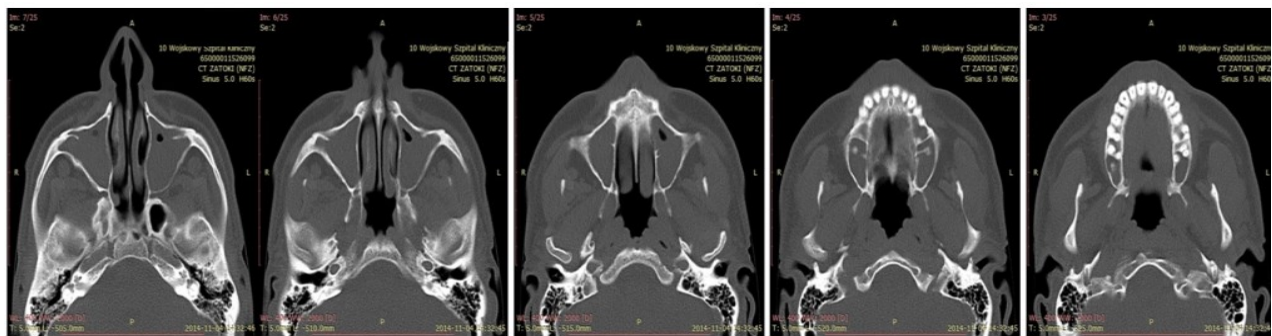

Fig. 4. The use of computed tomography images to create a 3D model for printing from polymer materials

Also for illustrative purposes and verification of the possibility of performing a complicated surgical procedure, a 3D model of the upper spine was created based on the data of a particular patient (fig. 5). In this particular case, it was about the indication of optimal geometry and mechanical reinforcement of the weakened part of the spine.

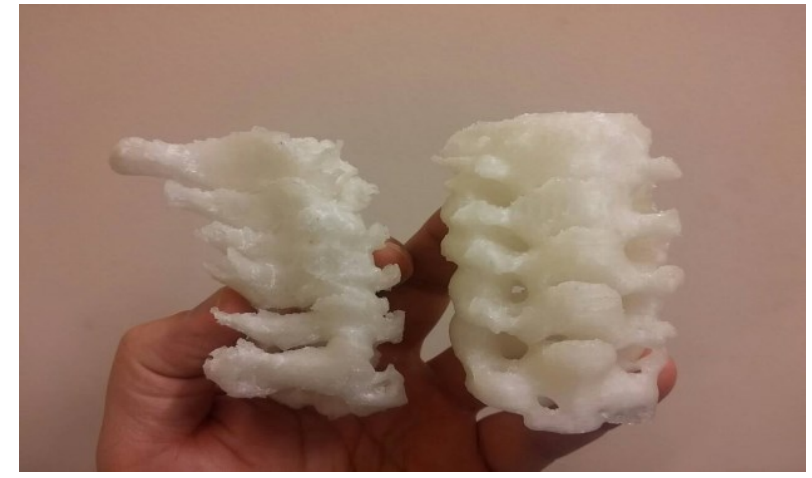

Fig. 5. The model 3D of the spine printed from polymers

The aim of the next work was to create training organs for surgeons of urologists, so that on their basis it was possible to conduct the training of connecting the ure-ter with the bladder after removal of the prostate gland. The manufactured organs were evaluated by specialists. The figure shows the components that were used to conduct the trial training and the view of the training station (Fig. 6).
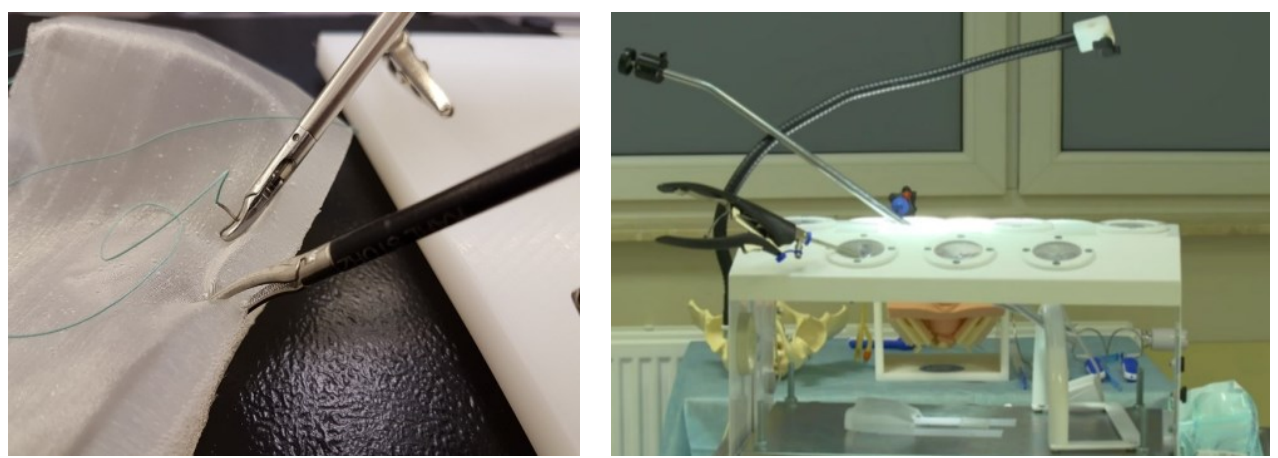

Fig. 6. The view of artificial organs and a method of joining artificial polymer organs and the view of training station 


\section{Summary and conclusions}

Based on the work in the field of the manufacturing artificial 3D organs for training purposes, it can be concluded that they lead to an increase in the quality of real surgical operations but also increase the quality of the models produced. Current concepts of manufacturing 3D medical organs offer various possibilities for both scientists and doctors, which will lead to easier development of standard solutions. In addition, developing a production concept for personalized anatomical models can be one of the milestones in the direction of printing ondemand an individualized body. The possibility of using 3D models from polymers in the field of laryngology, urology and neurosurgery was verified positively. Further research into the editing and production of artificial organs aims to develop technologies that provide remote access, file exchange and processing with the required level of data security, simultaneously printed from various materials, shaping the properties of new technologies useful in everyday clinical practice, awareness and education of medical staff and patients, cooperation in interdisciplinary treatment by the team, new models of rehabilitation and care with patients, implants, 3D printing. The presented concept of 3D models production is a relatively new solution, but its further development may lead to the emergence of a new family of commercial initiatives aimed at combining common efforts aimed at optimization based on 3D technology in everyday clinical practice.

\section{References}

1. LE Copes, LM Lucas, JO Thostenson, HE Hoekstra, DM Boyer, A collection of nonhuman primate computed tomography scans housed in MorphoSource, a repository for $3 D$ data. Sci Data 3/160001 (2016)

2. YE Choonara, LC du Toit, P. Kumar, PP Kondiah, V Pillay, 3D-printing and the effect on medical costs: a new era? Expert Rev Pharmacoecon Outcomes; 16, p.23-32 (2016)

3. M. Macko, Z. Szczepański, D. Mikołajewski, E. Mikołajewska, S. Listopadzki, The Method of Artificial Organs Fabrication Based on Reverse Engineering in Medicine. Lecture Notes in Mechanical Engineering. Book ID: 419988_1_En. Springer International Publishing AG. E. Rusinski and D. Pietrusiak (eds.) (2017)

4. M. Macko, Z. Szczepański, E. Mikołajewska, J. Nowak, D. Mikolajewski, Repository of $3 D$ images for education and everyday clinical practice purposes. Article in BioAlgorithms and Med-Systems 13(2), 01 (2017)

5. E. Mikołajewska, M. Macko, D. Mikołajewski, Ł. Ziarnecki, S. Stańczak, P. Kawalec, Medical and military applications of $3 D$ printing. J. of Sc. of the gen. Tadeusz Kosciuszko Military Academy of Land Forces;179:128-41(2016)

6. M. Macko, E. Mikołajewska, Z. Szczepański, B. Augustyńska, D. Mikołajewski, Repository of images for reverse engineering and medical simulation purposes. Med Biol Sci; 30:23-9 (2016)

7. K. Pepliński, P. Czyżewski, D. Górecki, D. Sykutera, M. Bieliński, Selected geometrical features and strength indicators of elements manufactured by fused deposition modeling technology, 01 Polymers (2017)

8. D. Radenkovic, A. Solouk, A. Seifalian, Personalized development of human organs using 3D printing technology, Med Hypotheses, 87:30-3 (2016)

9. W. Shui, M. Zhou, S. Chen, Z. Pan, Q. Deng, Y. Yao, et al. The production of digital and printed resources from multiple modalities using visualization and threedimensional printing techniques. Int J Comput Assist Radiol Surg (2016)

10. Y. Gür, Additive manufacturing of anatomical models from computed tomography scan data. Mol Cell Biomech, 11, 249-58 (2014)

11. Y. He, GH Xue, JZ Fu, Fabrication of low cost soft tissue prostheses with the desktop 3D printer. Sci Rep, 4:6973 (2014) 
12. J. Iwaniec, M. Iwaniec, Heart work analysis by means of recurrence-based methods, Diagnostyka Open Access Volume 18, Issue 4, p. 89-96 (2017)

13. M. Kajor, D. Grochala, M. Iwaniec, E. Kantoch, D. Kucharski, A prototype of the mobile stethoscope for telemedical application, 14th International Conference on Perspective Technologies and Methods in MEMS Design, MEMSTECH, Lviv; Ukraine; CFP1864A-PRT; Code 136746, p. 5-18 (2018)

14. N. Jaworski, M. Iwaniec, Numerical simulation of dental implant insertion process, 13th International Conference Perspective Technologies and Methods in MEMS Design, MEMSTECH, Lviv, Ukraine, CFP1744A-PRT; Code 128152, p. 59-61 (2017) 